\title{
TRISTEZA MATERNA EM PUÉRPERAS E FATORES ASSOCIADOS
}

| Marcela de Andrade Pereira Silva ${ }^{1}$; Marcela Demitto²; Cátia Agnolo ${ }^{3}$; Maricy Torres ${ }^{4}$; Maria Carvalho ${ }^{5}$; Sandra Pelloso ${ }^{6}$

\section{RESUMO}

CONTEXTO: A gravidez e a maternidade são importantes na vida das mulheres, mas podem gerar alterações físicas e emocionais tornando-as vulneráveis.

OBJETIVO: Analisar a presença de sintomas de tristeza materna vivenciados por puérperas e seus fatores associados.

MÉTODOS: Trata-se de um estudo transversal, quantitativo, realizado com 278 mulheres internadas em uma Maternidade de um município do Noroeste do Paraná, Sul do Brasil, entre janeiro a junho de 2015. Foram utilizadas a Escala de Depressão de Edimburgo (EPDS) e a Escala de Humor Brasileira (BRAMS) para coleta de dados.

RESULTADOS: Escores $\geq 11$ na EPDS apresentaram-se associados estatisticamente à baixa condição econômica, multiparidade, gravidez não planejada, história de depressão e distúrbio do sono. Em relação à BRAMS, mulheres com renda per capta acima de 300,00 reais apresentaram sentimentos de vigor, entretanto, os sentimentos de raiva, depressão e fadiga, mostraram-se associados à multiparidade, gravidez não planejada, história de depressão e distúrbio do sono.

CONCLUSÕES: O alto percentual de mulheres que apresentaram sintomas depressivos justifica a importância e necessidade de cuidados com a saúde mental da mulher no período gestacional e puerperal, visto que são períodos de vulnerabilidade e transformação.

PALAVRAS-CHAVE: Saúde mental; Período pós-parto; Depressão Pós-parto; Epidemiologia

\section{RESUMEN}

"Depresión posparto en mujeres en el postparto y factores asociados"

CONTEXTO: El embarazo y la maternidad son importantes en la vida de las mujeres, pero pueden causar cambios físicos y emocionales que los hace vulnerables.

OBJETIVO: Analizar la presencia de síntomas de la depresión posparto experimentadas por las madres y sus factores asociados. MÉTODOS: Se trata de un estudio transversal, cuantitativo con 278 mujeres internadas en una maternidad en una ciudad del noroeste, Paraná, sur de Brasil, de enero a junio de 2015. Se utilizó la Escala de Depresión de Edimburgo (EPDS) y Escala brasileña del estado de ánimo (BRAMS) para la recogida de datos.

RESULTADOS: Las puntuaciones $\geq 11$ en la EPDS se han asociado estadísticamente con bajo nivel económico, la multiparidad, los embarazos no planificados, una historia de depresión y los trastornos del sueño. En cuanto a BRAMS, las mujeres con ingresos per cápita de hasta 300,00 reales tenían sentimientos de vigor; Sin embargo, los sentimientos de ira, la depresión y la fatiga se asociaron con la multiparidad, los embarazos no planificados, una historia de depresión y los trastornos del sueño.

CONCLUSIONES: El alto porcentaje de las mujeres que tenían síntomas depresivos justifica la importancia y necesidad de preocuparse por la salud mental de las mujeres durante el embarazo y el puerperio, así como los períodos de vulnerabilidad y transformación.

DESCRIPTORES: Salud mental; Periodo posparto; Depresión posparto; Epidemiología

\begin{abstract}
"Maternity blues in postpartum women and associated factors"

BACKGROUND: Pregnancy and motherhood are important in women's lives, but they can generate physical and emotional changes making them vulnerable.
\end{abstract}

OBJECTIVE: To analyze the presence of maternal sadness symptoms experienced by postpartum women and their associated factors.

METHODS: This is a cross-sectional, quantitative study of 278 women admitted to a Maternity Hospital in the Northwest of Paraná, Southern Brazil, between January and June 2015. The Edinburgh Depression Scale (EPDS) and The Brazilian Humor Scale (BRAMS) for data collection.

RESULTS: Scores $\geq 11$ in the EPDS were statistically associated with low economic status, multiparity, unplanned pregnancy, history of depression and sleep disturbance. Regarding BRAMS, women with per capita income above $\mathrm{R} \$ 300.00$ reais showed feelings of vigor; however, feelings of anger, depression and fatigue were shown to be associated with multiparity, unplanned pregnancy, history of depression and sleep disturbance.

CONCLUSIONS: The high percentage of women who presented depressive symptoms justifies the importance and need of care for women's mental health in the gestational and puerperal periods, since they are periods of vulnerability and transformation.

\section{KEYWORDS: Mental health; Postpartum period; Postpartum depression; Epidemiology}

Submetido em 29-11-2016

Aceite em 11-10-2017

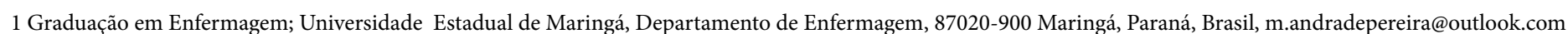

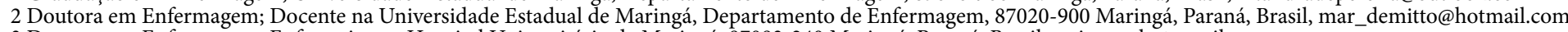
3 Doutora em Enfermagem; Enfermeira no Hospital Universitário de Maringá, 87083-240 Maringá, Paraná, Brasil, catiaagnolo@gmail.com

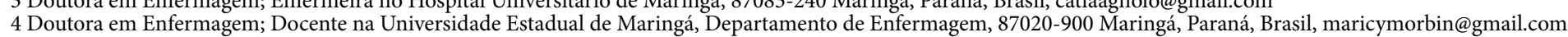

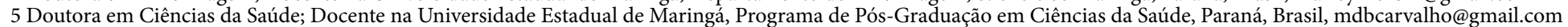

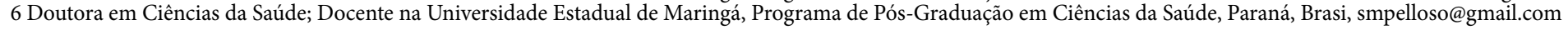

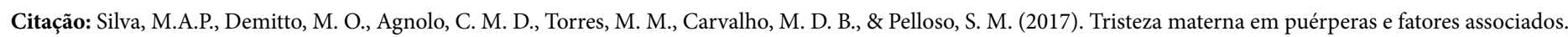
Revista Portuguesa de Enfermagem de Saúde Mental (18), 08-13. doi: 10.19131/rpesm.0186 


\section{INTRODUÇÃO}

A gravidez e a maternidade são eventos importantes na vida dos casais, mas podem gerar alterações físicas e emocionais tornando-os vulneráveis em alguns momentos. No período puerperal a mulher pode apresentar sintomas relacionados às alterações emocionais e estes se traduzem em transtornos mentais e comportamentais, entre eles a tristeza puerperal (Pereira e Rumel, 2007).

Tristeza puerperal, também conhecida como Maternity Blues ou Postpartum Blues é encontrada no CID-10 subgrupo F53, e caracteriza-se por choro, irritabilidade, rápidas mudanças de humor (Kaplan \& Sadock, 1999) e não há consenso quanto à possibilidade ou não de humor deprimido (Cox, Holden \& Sagovsky, 1987). É considerado como uma perturbação de humor, mas há pouca conformidade na sua definição (Goyal, Gay \& Lee, 2010). A incidência de Maternity Blues varia de 50 a $80 \%$ e geralmente aparece entre o $3 .^{\circ}$ e $10 .^{\circ}$ dia após o parto, é raro, mas a condição pode evoluir para um transtorno severo (Kaplan \& Sadock, 1999), sendo também um fator de risco para a depressão pós-parto (Austin, Kildea \& Sullivan, 2007).

Nos Estados Unidos 26-85\% das mães apresentam alteração de humor no pós-parto (Goyal, Gay \& Lee, 2010). Em estudo comparativo entre mulheres residentes na Coréia e outros países asiáticos a incidência variou de 23\% a 36,1- 48\% (Chung, Yoo \& Joung, 2013).

Em estudo realizado com 70 mulheres no Sul do Brasil os autores encontraram $67,2 \%$ das mulheres com a presença de tristeza no pós-parto imediato. Dentre elas, $8,6 \%$ apresentaram o sintoma todos os dias, durante todo o dia; $31,5 \%$ de mulheres que relataram a presença de tristeza também todos os dias, mas em determinados momentos; e $27,1 \%$ a ocorrência de tristeza em momentos casuais (Pereira \& Rumel, 2007).

Atualmente a depressão pós-parto é considerada uma epidemia silenciosa, uma vez que a identificação dos sintomas é tardia. Aproximadamente 50\% das mulheres com depressão pós-parto não é devidamente diagnosticada porque os profissionais de saúde ainda desconhecem e não sabem avaliar os sintomas depressivos (Wisner, Chambersa \& Sit, 2006).

Estudo do Sul do Brasil identificou que 77,8\% das mulheres que vivenciaram doença mental, apresentaram o sintoma de tristeza no pós-parto imediato, porém o sintoma também foi encontrado em $50 \%$ das mulheres que nunca vivenciaram uma doença mental (Pereira \& Rumel, 2007).
Até onde sabemos, existe uma escassez de estudos envolvendo mães logo após o nascimento de seu filho com ou sem depressão pós-parto. Existem características relacionadas à tristeza puerperal que necessitam ser discutidos, principalmente em relação à triagem para o estado de humor materno na primeira semana após o parto que é o período mais importante para prevenir a depressão pós-parto (Dennis, 2004).

Nesse sentido, o objetivo deste estudo foi analisar a presença de sintomas de Maternity Blues vivenciado por mulheres no puerpério imediato e correlacioná-los com fatores sociodemográficos, obstétricos e transtornos mentais prévios.

\section{MÉTODOS}

Trata-se de um estudo transversal e quantitativo realizado com 278 puérperas no puerpério mediato, internadas em uma Maternidade de um município do Sul do Brasil, valor proporcional ao número de nascidos vivos na Maternidade referida no ano de 2013 (3.466), com $95 \%$ de nível de confiança e 0,05 margem de erro. Os critérios de inclusão foram: puérperas internadas após 48 horas e antes da alta e de exclusão estiveram as puérperas com óbito fetal, aborto e recém-nascido internados na Unidade de Terapia Intensiva Neonatal. A coleta de dados foi realizada no período de Janeiro a Junho de 2015.

Como instrumento de coleta de dados foi utilizado um questionário estruturado, com questões sóciodemograficas, obstétricas e referentes à história prévia de distúrbios mentais, a Escala de Depressão de Edimburgo (Edinburgh Postnatal Depression Scale - EPDS) e a Escala de Humor Brasileira (BRAMS).

Escala de Depressão de Edimburgo, foi adaptada e validada em diversos países, incluindo o Brasil, é autoaplicável, com dez itens, divididos em quatro graduações ( 0 a 3) e mede a presença e intensidade de sintomas depressivos nos últimos sete dias (Figueira, Corrêa, Malloy-Diniz \& Romano-Silva, 2009). Já a Escala de Humor Brasileira (BRAMS), é a versão brasileira da Escala de Humor de Brunel (BRUMS), foi validada para a população brasileira (Rohlfs, 2006; Terry, Lane \& Fogarty, 2003) e permiti uma rápida mensuração do estado de humor de populações compostas por adultos e adolescentes (Rohlfs, 2006).

Após, a coleta dos dados, os mesmos foram transcritos para a planilha do programa Microsoft Office Excel $2010^{\circledR}$ e submetidos à análise estatística, utilizado o programa Statistical Package for Social Sciences (SPSS). 
O estudo obedeceu a todos os princípios éticos e legais envolvendo pesquisa com seres humanos, com parecer positivo (n. 852.156) do Comitê de Ética em Pesquisa da Universidade Estadual de Maringá.

\section{RESULTADOS}

Participaram da pesquisa 278 mulheres no puerpério imediato. Os escores da Escala de Depressão de Edimburgo iguais ou superiores a 11, ponto de corte estabelecido por Santos, Martins \& Pasquali (1999), o qual descreve a presença de sintomas depressivos, e os fatores associados podem ser vistos na Tabela 1 .

Tabela 1 -Escala de Depressão de Edimburgo (EPDS) e Fatores Associados

\begin{tabular}{|c|c|c|c|c|}
\hline & $\mathbf{n}$ & $\%$ & EPDS $>11$ & Valor $\mathbf{p}$ \\
\hline \multicolumn{3}{|l|}{ Idade (anos) } & $\mathrm{n}(\%)$ & \\
\hline$\leq 19$ & 49 & 17,6 & $9(18,4)$ & 0,09 \\
\hline $20-34$ & 196 & 70,5 & $40(20,4)$ & \\
\hline$\geq 35$ & 33 & 11,9 & $12(36,4)$ & \\
\hline \multicolumn{5}{|l|}{ Estado civil } \\
\hline Com companheiro & 121 & 43,5 & $43(19,1)$ & \\
\hline Sem companheiro & 47 & 16,9 & $18(33,9)$ & 0,01 \\
\hline \multicolumn{5}{|l|}{ Escolaridade } \\
\hline$<8$ anos & 43 & 15,5 & $14(32,6)$ & 0,06 \\
\hline$\geq 8$ anos & 235 & 84,5 & $47(20,0)$ & \\
\hline \multicolumn{5}{|l|}{ Paridade } \\
\hline Primípara & 126 & 45,3 & $20(15,9)$ & 0,02 \\
\hline Multípara & 152 & 54,7 & $41(26,9)$ & \\
\hline \multicolumn{5}{|l|}{ Aborto } \\
\hline Sim & 58 & 20,9 & $18(31,0)$ & 0,06 \\
\hline Não & 220 & 79,1 & $43(19,5)$ & \\
\hline \multicolumn{5}{|l|}{ Gravidez planejada } \\
\hline $\operatorname{Sim}$ & 106 & 38,1 & $16(15,1)$ & \\
\hline Não & 172 & 61,9 & $45(26,2)$ & 0,03 \\
\hline \multicolumn{5}{|l|}{ História de depressão } \\
\hline $\operatorname{Sim}$ & 40 & 14,4 & $18(45,0)$ & $<0,001$ \\
\hline Não & 238 & 85,6 & $43(18,1)$ & \\
\hline \multicolumn{5}{|l|}{ Distúrbio do Sono } \\
\hline Sim & 30 & 10,8 & $15(50,0)$ & $<0,001$ \\
\hline Não & 248 & 89,2 & $46(18,5)$ & \\
\hline
\end{tabular}

Houve associação estatística significativa quanto à presença de fatores depressivos em mulheres com renda per capta inferior ou igual a 150,00 reais, multiparidade, planejamento gestacional não realizado e história prévia de depressão e distúrbio do sono (Tabela n. ${ }^{\circ} 1$ ). Quanto à aplicação da Escala de Humor Brasileira, os resultados apresentados foram importantes, dentre eles, podemos citar o elevado percentual obtido na subescala vigor (98,9\%), um dado importante no caráter positivo. Entretanto, as subescalas que representam sentimentos pouco favoráveis no período puerperal, também apresentaram percentuais elevados, sendo eles, $88,1 \%$ (fadiga), $82 \%$ (tensão), 56,8\% (confusão), 39,2\% (raiva) e 30,2\% (depressão). Estes valores e as associações estatísticas estão mostrados na Tabela 2 .

Tabela 2 -Escala de Humor Brasileira e Fatores Associados

\begin{tabular}{|c|c|c|c|c|c|c|}
\hline & Raiva & $\begin{array}{l}\text { De- } \\
\text { pressão }\end{array}$ & $\begin{array}{l}\text { Con- } \\
\text { fusão }\end{array}$ & Fadiga & Tensão & Vigor \\
\hline Variáveis & $\mathrm{n}(\%)$ & $\mathrm{n}(\%)$ & $\mathrm{n}(\%)$ & $\mathrm{n}(\%)$ & $\mathrm{n}(\%)$ & $\mathrm{n}(\%)$ \\
\hline \multicolumn{7}{|l|}{ Idade (anos) } \\
\hline$\leq 19$ & $15(30,6)$ & $12(24,4)$ & $27(55,1)$ & $39(79,5)$ & $40(81,6)$ & $49(100)$ \\
\hline $20-34$ & $79(40,3)$ & $50(30,1)$ & $\begin{array}{l}115 \\
(58,6)\end{array}$ & $\begin{array}{l}174 \\
(88,7)\end{array}$ & $\begin{array}{l}160 \\
(81,6)\end{array}$ & $\begin{array}{l}193 \\
(98,4)\end{array}$ \\
\hline$\geq 35$ & $15(45,5)$ & $13(39,4)$ & $16(48,4)$ & $32(96,9)$ & $28(84,8)$ & $33(100)$ \\
\hline Valor $\mathrm{p}$ & 0,33 & 0,35 & 0,53 & 0,05 & 0,9 & 0,53 \\
\hline \multicolumn{7}{|l|}{ Estado civil } \\
\hline $\begin{array}{l}\text { Com com- } \\
\text { panheiro }\end{array}$ & $86(38,2)$ & $64(28,4)$ & $\begin{array}{l}126 \\
(56,0)\end{array}$ & $\begin{array}{l}200 \\
(88,9)\end{array}$ & $\begin{array}{l}178 \\
(79,1)\end{array}$ & $\begin{array}{l}223 \\
(99,1)\end{array}$ \\
\hline $\begin{array}{l}\text { Sem com- } \\
\text { panheiro }\end{array}$ & $23(43,4)$ & $20(37,7)$ & $32(60,4)$ & $45(84,9)$ & $50(94,3)$ & $\begin{array}{l}52 \\
(84,9)\end{array}$ \\
\hline Valor $\mathrm{p}$ & 0,48 & 0,18 & 0,56 & 0,41 & 0,009 & 0,52 \\
\hline \multicolumn{7}{|c|}{ Escolaridade } \\
\hline$<8$ anos & $17(39,5)$ & $14(32,5)$ & $27(62,7)$ & $37(86,0)$ & $36(83,7)$ & $43(100)$ \\
\hline$\geq 8$ anos & $92(39,1)$ & $70(29,7)$ & $\begin{array}{l}131 \\
(55,7)\end{array}$ & $\begin{array}{l}208 \\
(88,5)\end{array}$ & $\begin{array}{l}192 \\
(81,7)\end{array}$ & $\begin{array}{l}232 \\
(98,7)\end{array}$ \\
\hline Valor $\mathrm{p}$ & 0,96 & 0,71 & 0,39 & 0,64 & 0,75 & 0,6 \\
\hline \multicolumn{7}{|l|}{ Paridade } \\
\hline Primípara & $20(15,9)$ & $28(22,2)$ & $77(61,1)$ & $\begin{array}{l}105 \\
(83,3)\end{array}$ & $\begin{array}{l}102 \\
(80,9)\end{array}$ & $\begin{array}{l}125 \\
(99,2)\end{array}$ \\
\hline Multípara & $41(26,9)$ & $56(36,8)$ & $81(53,2)$ & $\begin{array}{l}140 \\
(92,1)\end{array}$ & $\begin{array}{l}126 \\
(82,8)\end{array}$ & $\begin{array}{l}150 \\
(98,6)\end{array}$ \\
\hline Valor $\mathrm{p}$ & 0,02 & $<0,01$ & 0,18 & 0,02 & 0,67 & 0,57 \\
\hline \multicolumn{7}{|l|}{ Aborto } \\
\hline Sim & $26(44,8)$ & $19(32,7)$ & $36(62,0)$ & $49(84,4)$ & $49(84,4)$ & $\begin{array}{l}57 \\
(98,2)\end{array}$ \\
\hline Não & $83(37,7)$ & $65(29,5)$ & $\begin{array}{l}122 \\
(55,4)\end{array}$ & $\begin{array}{l}196 \\
(89,0)\end{array}$ & $\begin{array}{l}179 \\
(81,3)\end{array}$ & $\begin{array}{l}218 \\
(99,0)\end{array}$ \\
\hline Valor $\mathrm{p}$ & 0,32 & 0,63 & 0,36 & 0,33 & 0,58 & 0,5 \\
\hline \multicolumn{7}{|c|}{ Gravidez planejada } \\
\hline Sim & $16(15,0)$ & $26(24,5)$ & $58(54,7)$ & $92(86,7)$ & $86(81,1)$ & $\begin{array}{l}105 \\
(99,0)\end{array}$ \\
\hline Não & $45(26,1)$ & $58(33,7)$ & $\begin{array}{l}100 \\
(58,1)\end{array}$ & $\begin{array}{l}153 \\
(88,9)\end{array}$ & $\begin{array}{l}142 \\
(82,5)\end{array}$ & $\begin{array}{l}170 \\
(98,8)\end{array}$ \\
\hline Valor $\mathrm{p}$ & 0,03 & 0,1 & 0,57 & 0,58 & 0,76 & 0,67 \\
\hline \multicolumn{7}{|c|}{ História de depressão } \\
\hline Sim & $21(52,5)$ & $20(50,0)$ & $28(70,0)$ & $37(92,5)$ & $35(87,5)$ & $40(100)$ \\
\hline Não & $88(36,9)$ & $64(26,8)$ & $\begin{array}{l}130 \\
(54,6)\end{array}$ & $\begin{array}{l}208 \\
(87,3)\end{array}$ & $\begin{array}{l}193 \\
(81,0)\end{array}$ & $\begin{array}{l}235 \\
(98,7)\end{array}$ \\
\hline Valor $\mathrm{p}$ & 0,06 & 0,003 & 0,06 & 0,35 & 0,32 & 0,62 \\
\hline \multicolumn{7}{|c|}{ Distúrbio de Sono } \\
\hline Sim & $15(50,0)$ & $14(46,6)$ & $21(70,0)$ & $27(90,0)$ & $27(90,0)$ & $30(100)$ \\
\hline Não & $46(18,5)$ & $70(28,2)$ & $\begin{array}{l}137 \\
(55,2)\end{array}$ & $\begin{array}{l}218 \\
(87,9)\end{array}$ & $\begin{array}{l}201 \\
(81,5)\end{array}$ & $\begin{array}{l}245 \\
(98,7)\end{array}$ \\
\hline Valor $\mathrm{p}$ & $<0,001$ & 0,03 & 0,12 & 0,51 & 0,22 & 0,7 \\
\hline
\end{tabular}




\section{DISCUSSÃO}

Os transtornos psiquiátricos no puerpério implicam na relação mãe-filho gerando consequências graves, visto que a instabilidade emocional da mãe impossibilita uma maior interação com seu filho (Moura, Fernandes e Apolinário, 2011).

Estudos envolvendo mulheres no puerpério são fundamentais para o levantamento de informações e possíveis fatores de risco para transtornos de humor comuns no período puerperal, possibilitando o planejamento de ações preventivas e precoces dessas alterações, afim de minimizar os danos ao binômio mãe-filho. Além de reforçar a importância e necessidade de cuidados com a saúde mental da mulher no período gestacional e puerperal, visto que são períodos de vulnerabilidade e transformação.

Os resultados de um estudo realizado com puérperas em Camarões, sugerem que o espaço de discurso pósparto, incluindo a qualidade das respostas emocionais do cuidador, determinam a gestão da depressão pósparto e limita a sua descompensação (Mboua, Nkoum \& Abessouguié, 2016).

A prevalência de mulheres que apresentaram sintomatologia depressiva de acordo com a Escala de Depressão de Edimburgo foi de 21,9\%. Em estudos nacionais a prevalência variou de $11-24,2 \%$ (Gomes et al., 2010; Menezes et al., 2012), já em estudo internacional a percentagem foi de 19,2\% (Masmoudi et al., 2014).

Outro estudo realizado com mulheres até 2 semanas após o parto, concluiu que a morbidade psiquiátrica é muito comum no período pós-operatório, podendo ocorrer logo na primeira semana após o parto. Neste estudo a porcentagem de alterações psiquiátricas encontradas foi de $44 \%$, sendo que o distúrbio mais frequente foi a depressão, presente em $26 \%$ das mulheres (Kumar, Nagaraj, Koudike \& Maigi, 2016).

Outro estudo constatou a presença de $7 \%$ de mulheres deprimidas (EPDS $>=11$ ), onde um episódio prévio de depressão, fatos relevantes da vida e parto instrumental foram independentemente associados à depressão (Pop et al., 2016).

O percentual significativo de puérperas que apresentaram no período puerperal imediato sintomas depressivos enfatiza a necessidade e importância da capacitação dos profissionais de saúde em realizar uma avaliação do estado de humor da puérpera precocemente (Menezes et al., 2012), visto que, sintomas de sofrimento mental não reconhecidos ou não valorizados podem resultar em repercussões imediatas e/ou tardias para a puérpera e o recém nascido (Brito, Alves, Ludermir, \& Araújo, 2015).
Quanto aos fatores associados aos sintomas depressivos, verificou-se que a situação econômica baixa e a história prévia de depressão, foram relevantes na presença de sintomas depressivos, resultado também encontrado em estudo realizado na Tunísia com 302 puérperas entrevistadas na primeira semana puerperal, no qual, a obtenção de um escore significativo na Escala de Depressão de Edimburgo $(\geq 10)$ se mostraram associadas ao baixo nível socioeconômico e a presença de antecedentes psiquiátricos (Masmoudi et al., 2014). Em outro estudo realizado com 70 mulheres no Hospital Nossa Senhora da Conceição em Santa Catarina, mostrou que $77,8 \%$ das mulheres que vivenciaram doença mental previamente, apresentaram o sintoma tristeza no pós-parto imediato. Mesmo que não associado estatisticamente o valor foi superior ao número de mulheres que apresentaram o sintoma tristeza e não possuíam essa vivencia prévia (50\%) (Pereira e Rumel, 2007).

O fator socioeconômico também teve associação estatística quando aplicado a Escala de Humor Brasileira, no qual, os resultados mostraram que mulheres com melhores condições econômicas tenderam a apresentar sentimentos de vigor e mulheres com uma condição econômica baixa apresentaram em maior número sentimentos depressivos.

Mulheres com história prévia de depressão e distúrbio do sono tenderam a apresentar sintomas de raiva e depressão presentes na Escala de Humor Brasileira, apresentando associação estatística significativa.

Outro fator que se mostrou associado à presença de sintomas depressivos foi o não planejamento gestacional. Estudo realizado em Recife (PE) com 1.057 mulheres entrevistadas após o parto, mostrou que mulheres com gravidez não pretendida tiveram maior chance de apresentar sentimentos depressivos durante o período pósparto (Brito, et al., 2015).

Esse fator também teve associação com sentimentos de raiva e depressão apresentados pela Escala de Humor Brasileira, no qual, 26,1 das mulheres que tiveram uma gravidez não pretendida apresentaram sentimentos de raiva e $33,7 \%$, depressão.

Para prevenir uma gravidez inoportuna e consequentemente minimizar a chance de desenvolver algum distúrbio de humor no pós-parto, cabe ao sistema de saúde, principalmente em âmbito de atenção primária, enfatizar e orientar de forma precisa e adequada o uso de métodos contraceptivos masculinos e femininos, além da oferta dos mesmos. 
O fator multiparidade teve associação estatística com a presença de sintomas depressivos, no qual, 54,7\% das puérperas multíparas tiveram um escore $\geq 11$ na Escala de Depressão de Edimburgo, configurando um episódio depressivo, na Escala de Humor Brasileira esse mesmo fator teve associação com a presença de sintomas de raiva, depressão e fadiga.

Estudo realizado na região Sul do Estado do Rio Grande do sul com 53 puérperas internadas na maternidade do Hospital Universitário da região, evidenciou uma susceptibilidade em desenvolver depressão pós-parto em mulheres multíparas (Menezes et al., 2012).

\section{CONCLUSÃO}

Os resultados obtidos mostraram um elevado percentual de mulheres susceptíveis a desenvolver um transtorno de humor puerperal, reforçando a importância e necessidade de cuidados com a saúde mental da mulher no período gestacional e puerperal, visto que são períodos de vulnerabilidade e transformação.

\section{IMPLICAÇÕES PARA A PRÁTICA CLÍNICA}

Diversos fatores se mostraram com risco para a presença de sintomas depressivos no período puerperal, sendo de grande importância no diagnóstico precoce e no planejamento de ações preventivas. Para tanto, percebe-se a necessidade de capacitações da equipe multiprofissional, para triagem adequada e identificação precoce de sintomas depressivos, podendo interver de forma hábil, minimizando as consequências negativas do transtorno mental vivenciadas durante o período puerperal.

Instrumentos que auxiliam esse rastreamento são de grande significância, e a escala de depressão de Edimburgo e de Humor Brasileira se mostraram eficazes e práticas.

\section{REFERÊNCIAS BIBLIOGRÁFICAS}

Austin, M. P., Kildea, S., \& Sullivan, E. (2007). Maternal mortality and psychiatric morbidity in the perinatal period: Challenges and opportunities for prevention in the Australian setting. Medical Journal of Australia, 186, 364-367.

Brito, C. N. O., Alves, V. A., Ludermir, A. B., e Araújo, T. V. B. A. (2015). Depressão pós-parto entre mulheres com gravidez não pretendida. Revista de Saúde Pública, 49, 33. doi: 10.1590/S0034-8910.2015049005257 
Chung, S.S., Yoo, I. Y., \& Joung, K. H. (2013). Postpartum blues among Korean mothers: A structural equation modelling approach. International Journal of Mental Health Nursing, 22, 359-367. doi: 10.1111lj.1447-0349.2012.00880.x

Cox, J. L., Holden, J. M., \& Sagovsky, R. (1987). Detection of postnatal depression. Development of the 10item Edinburgh Postnatal Depression Scale. British Journal of Psychiatry, 150, 782-786.

Dennis, C. L. (2004). Can we identify mothers at risk for postpartum depression in the immediate postpartum period using the Edinburgh Postnatal Depression Scale?. Journal of Affective Disorders, 78, 163-169.

Figueira, P., Corrêa, H., Malloy-Diniz, L., e RomanoSilva, M. A. (2009). Edimburgo pós-natal Depression Scale para triagem no sistema público de saúde. Revista de Saúde Pública, 43(Suppl. 1), 79-84. doi: 10.1590/ S0034-89102009000800012

Gomes, L. A., Torquato V. S., Feitoza, A. R., de Souza, A. R., da Silva, M. A. M., e Pontes, R. J. (2010). Identificação dos fatores de risco para depressão pós parto: Importância do diagnóstico precoce. Revista da Rede de Enfermagem do Nordeste, 11, 117-123.

Goyal, D., Gay, C., \& Lee, K. A. (2010). How much does low socioeconomic status increase the risk of prenatal and postpartum depressive symptoms in first-time mothers?. Women's Health Issues, 20, 96-104.

Kaplan, H. I., \& Sadock, B. J. (1999). Tratado de Psiquiatria. Porto Alegre: Artmed.

Kumar, N., Nagaraj, A. K. M., Koudike, U., \& Majgi, S. M. (2016). Psychiatric morbidity and correlates in postpartum women in a tertiary care hospital. Indian Journal of Psychological Medicine. 38, 309-314. doi: 10.4103/0253-7176.185956

Masmoudi, J., Charfeddine, F., Travelsi, S., Ven Ayad, B., Guermazi, M., Baâti, I., \& Jaoua, A (2014). Postpartum depression: Prevalence and risk factors. A prospective study concerning 302 tunisian parturients. La tunisie Medicali, 92, 615-621.
Mboua, C. P., Nkoum, B. A., \& Abessouguié, S. P. (2016). La dimension du soin relationnel dans la décompensation du blues en post-partum intermédiaire au Cameroun. Médecine et Santé Tropicales, 26, 273 277. doi: 10.1684/mst.2016.0577

Menezes, F. L., Netto de Oliveira, A. M., Pintos Lemos, L. A., da Silva, P. A., Kerber, P. C., \& da Silva, M. R. S. (2012). Frequência da depressão puerperal na maternidade de um hospital universitário da Região Sul. Revista Electrónica Trimestral de Enfermería, 27, 419-429.

Moura, E. C. C., Fernandes, M. A., e Apolinário, F. I. R. (2011). Percepção materna sobre transtornos psiquiátricos no puerpério: Implicações na relação mãe-filho. Revista Brasileira de Enfermagem, 64, 445450 .

Pereira, C. W., e Rumel, D. (2007). Epidemiologia da tristeza em puérperas no pós-parto imediato. Femina, $35,369-377$.

Pop, V. J. M., Truijens, S. E. M., Spek, V., Wijnen, H. A., van Son, M. J. M., \& Bergink, V. (2016). A new concept of maternity blues: Is there a subgroup of women with rapid cycling mood symptoms?. Journal of Affective Disorders, 177, 74-79. doi: 10.1016/j.jad.2015.02.015

Rohlfs, I. C. M. (2006). Validação do Teste Brums para avaliação de humor em atletas e não atletas brasileiros (Dissertação de Mestrado). Universidade do Estado de Santa Catarina, Centro de Educação Física, Fisioterapia e Desportos.

Santos, M. F., Martins, F. C., e Pasqual, L. (1999). Escalas de auto-avaliação de depressão pós-parto: Estudo no Brasil. Revista de Psiquiatria Clínica, 26, 90-95.

Terry, P .C., Lane, A. M., \& Fogarty, G. J. (2003). Construct validity of the POMS-A for use with adults. Psychology of Sport and Exercise, 4, 125-139. doi: 10.1016/ S1469-0292(01)00035-8

Wisner, K., Chambers, C., \& Sit, D. K. Y. (2006). Postpartum depression: A major public health problem. Journal of the American Medical Association, 6, 26162618. doi: 10.1001/jama.296.21.2616 